\title{
Can Mutational Analysis Be Used To Assist Structure Determination of Peptides?
}

\author{
Liudmila Voronina, Valeriu Scutelnic, Chiara Masellis, and Thomas R. Rizzo*(0) \\ Laboratoire de Chimie Physique Moléculaire, École Polytechnique Fédérale de Lausanne, EPFL SB ISIC LCPM, Station 6, CH-1015 \\ Lausanne, Switzerland
}

\section{Supporting Information}

\begin{abstract}
Mutational analysis is widely used to study the relationship between sequence and structure of proteins and peptides. It is often assumed that substituting a proline with another amino acid "locks" the peptide bond in the trans conformation, allowing only a subset of the initial molecular geometries to be observed. To test this assumption, we assess the result of substituting two prolines in the bradykinin sequence with alanine using field-asymmetric ion mobility spectrometry combined with cryogenic ion spectroscopy in the gas phase. While the structure of the mutant coincides with a part of the conformational space of the original peptide, the higher flexibility of the alanine backbone compared to proline allows it to access additional structures. We conclude that proline-to-nonproline substitutions are helpful to assign structures, but they should be used in conjunction with spectroscopic techniques that allow detailed comparison of the structures of the mutant and the native peptide.
\end{abstract}

Cubstituting an amino acid in a protein sequence with another residue is often referred to as mutational analysis. ${ }^{1}$ This procedure reveals how critical a certain amino acid is for the structure and function of a protein. In particular, it has been used to study the role of proline as the only canonical amino acid for which cis and trans isomers of the peptide bond are comparable in energy. ${ }^{2}$ To assess the role of rare cis states (5.2\% according to Weiss and co-workers), ${ }^{3}$ the possibility of isomerization can be eliminated by "locking" the Xxx-Pro bond to the trans isomer via substituting the proline with another amino acid. ${ }^{4,5}$

Mutational analysis, in conjunction with various methods of structural determination, shows that proline performs multiple functions within the protein sequence: It acts as a molecular switch, ${ }^{6,7}$ and its isomerization is often a rate-limiting step in protein folding. ${ }^{8,9}$ In order to deepen the understanding of the structural role of proline, one can isolate a model system from its native environment and apply powerful gas-phase tools that can be implemented in a conformer-selective way. For example, it was suggested that proline is one of the primary reasons for the formation of distinct conformational families of short peptides in the gas phase, resulting in multiple peaks in their collisional-cross section (CCS) distributions determined by ion mobility spectrometry (IMS). ${ }^{10-13}$ In order to establish a correspondence between the features in the CCS distribution and conformational preferences of prolyl-peptide bonds, the same point mutations as in solution-phase studies have been used. ${ }^{11,14-16}$ In the case of triply protonated bradykinin (BK), for instance, three conformational families in the gas phase were attributed to different cis-trans isomers using proline-toalanine substitutions. ${ }^{15,17}$

It has been determined that protein secondary structure is influenced by the presence of proline in two somewhat orthogonal ways. On the one hand, proline disrupts helix and $\beta$ sheet formation and increases the level of backbone disorder. ${ }^{18}$ On the other hand, it is conformationally restricted and makes the protein backbone more rigid. ${ }^{19}$ If proline is substituted by another amino acid, the resulting structure depends on the interplay between these factors. Neverthless, mutational analysis is often based on the assumption that upon "locking" a peptide bond in the trans conformation, only a subset of the initial molecular geometries should be observed. $1,4,7,15,17,20-22$ In this work we test this assumption for a mutant of bradykinin using a combination of ion mobility spectrometry and doubleresonance, cryogenic-ion spectroscopy in the gas phase.

The experimental procedure is similar to that used previously $^{23}$ and is described in the Supporting Information. Briefly, we generate gas-phase ions by electrospray, separate them into conformational families using field asymmetric ion mobility spectrometry (FAIMS), ${ }^{24}$ and inject them into a home-built spectrometer, where we perform spectroscopic studies at cryogenic temperatures. ${ }^{25}$

We have demonstrated previously that FAIMS allows us to partially separate several conformational families of $\mathrm{BK}^{3+}$ (Figure 1a). ${ }^{23}$ Using cryogenic-ion spectroscopy as a detector for a specific conformation, we observe that $\mathrm{BK}^{3+}$ forms at least three conformational families in the gas phase, which we denoted I, II, and III. ${ }^{23}$ In this study we focus on kinetically trapped conformational families I and II observed directly after ESI with low collisional activation and preserved upon FAIMS separation.

Normal bradykinin includes prolines in positions 2, 3 and 7, and the barriers for cis-trans isomerization are considered to be the origin of distinct conformational families in the gas phase. ${ }^{15,17}$ Here we explore the conformational space of triply protonated Pro3\&7 $\rightarrow$ Ala mutant of BK with proline in position 2. Pierson and co-workers suggested that this mutant reproduces the structure of conformational family $\mathrm{B}$ of native $\mathrm{BK}$, separated by the drift tube IMS. ${ }^{17}$

Received: October 23, 2017

Published: February 7, 2018 

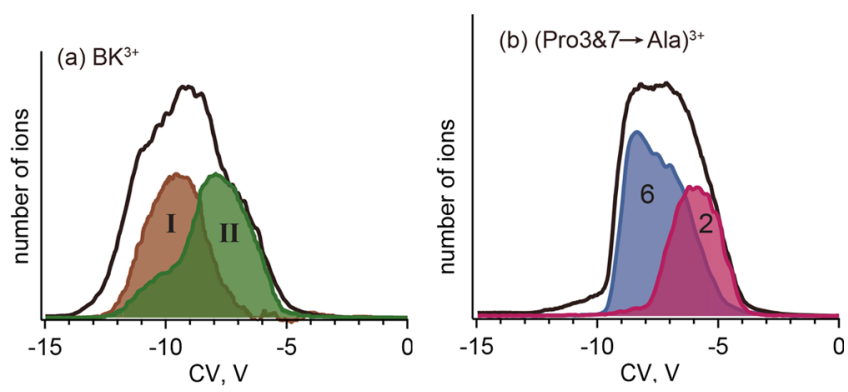

Figure 1. (a) $\mathrm{CV}$ distribution of $\mathrm{BK}^{3+}$ detecting all transmitted ions (black) and recorded via UV transitions unique for conformational families I and II. (b) CV distribution of Pro3\&7 $\rightarrow$ Ala mutant detecting all transmitted ions (black) and recorded via UV transitions 2 (pink) and 6 (blue) in Figure 2.

The CV distribution of this mutant in the gas phase (black trace, Figure $1 \mathrm{~b}$ ) exhibits maxima at $-8.2 \mathrm{~V}$ and $-7.1 \mathrm{~V}$. A part of it, which lies below $\sim-7 \mathrm{~V}$, overlaps with that of nonsubstituted bradykinin (Figure 1a), but significant ion signal appears at $\mathrm{CV}$ values between $-7 \mathrm{~V}$ to $-4 \mathrm{~V}$.

To distinguish the conformational families of Pro3\&7 $\rightarrow \mathrm{Ala}$, we set the $\mathrm{CV}$ on the shoulders of the distribution $(-8.3 \mathrm{~V}$ and $-6.0 \mathrm{~V}$ ) and record UV photofragmentation spectra (Figure 2). Three intense bands, labeled $1-3$, appear only at $\mathrm{CV}=-6.0 \mathrm{~V}$, while the band labeled 4 is unique to the photofragmentation spectrum recorded at $\mathrm{CV}=-8.3 \mathrm{~V}$.

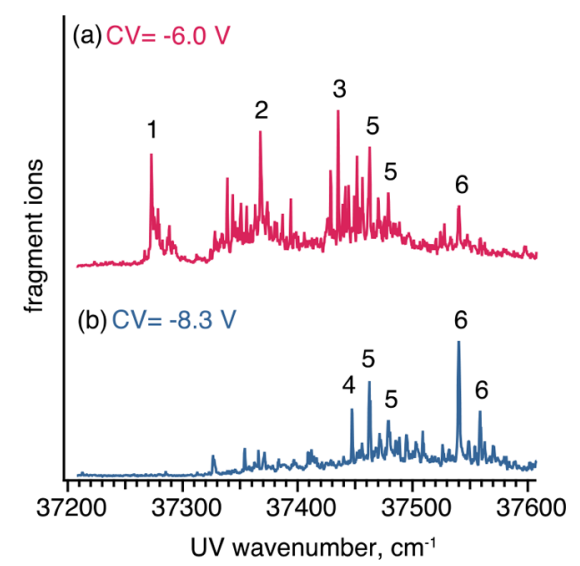

Figure 2. UV photofragmentation spectra of Pro3\&7 $\rightarrow$ Ala mutant with the $\mathrm{CV}$ value set to (a) $-6.0 \mathrm{~V}$ and (b) $-8.3 \mathrm{~V}$.

The CV distribution of the Pro3\&7 $\rightarrow$ Ala mutant can be separated into two main components by detecting selectively the photofragmentation signal on one absorption at a time (Figure $1 \mathrm{~b}$ ). The $\mathrm{CV}$ distributions corresponding to peaks 2 and 6 of the UV photofragment spectrum are shown; all others fall within one of the two presented. We thus observe that the conformational space of Pro3\& $\rightarrow \rightarrow$ Ala mutant consists of two main conformational families that appear at different characteristic compensation voltages.

The CV distributions of the Pro3\&7 $\rightarrow$ Ala mutant and nonsubstituted BK partially overlap. The maximum for conformational family II of BK lies at $\mathrm{CV}=-8.0 \mathrm{~V}$, while for the mutant we find a conformational family with a maximum at $\mathrm{CV}=-8.3 \mathrm{~V}$. To gain further insight into the relation between the structures of $\mathrm{BK}$ and the mutant, we compare the UV photofragmentation spectra of their over- lapping conformational families (Figure 3). The spectra are similar: two major bands of conformer 5 correspond to those of

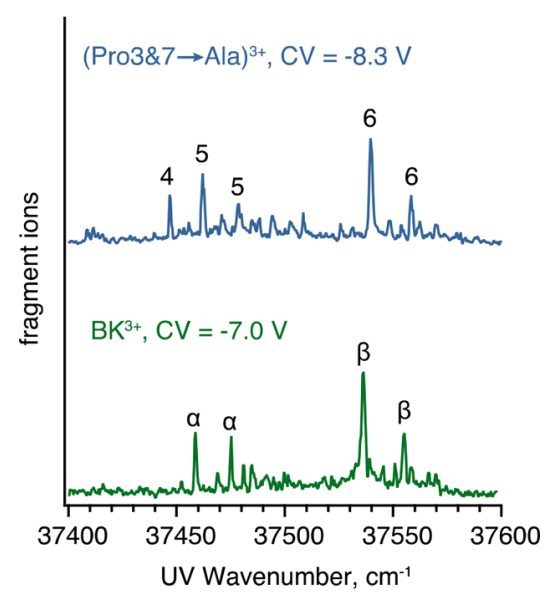

Figure 3. Comparison between UV photofragmentation spectra of Pro3\&7 $\rightarrow$ Ala mutant at $\mathrm{CV}=-8.3 \mathrm{~V}$ and conformational family II of $\mathrm{BK}^{3+}$ at $\mathrm{CV}=-7.0 \mathrm{~V}$. This $\mathrm{CV}$ value for $\mathrm{BK}$ is chosen to avoid spectral contamination from family I. The conformers identified by IR-UV double resonance spectroscopy are labeled.

conformer $\alpha$; the same holds for conformers 6 and $\beta$. However, a band appears in the photofragmentation spectrum of the mutant at $37439.2 \mathrm{~cm}^{-1}$, which corresponds to conformer 4 and is not present in the case of nonsubstituted BK.

The strongly similar pattern of UV bands shown in Figure 3 suggests that conformational family II of nonsubstituted BK is present among molecular geometries adopted by Pro3\&7 $\rightarrow$ Ala. At the same time, the mutant also forms conformer 4, which appears at the same $\mathrm{CV}$ as conformational family II of $\mathrm{BK}^{3+}$, but has a different structure. To confirm these conclusions, we compare the vibrational spectra of all conformers mentioned above (Figure 4). The spectra of conformers 6 of the mutant and $\beta$ of $\mathrm{BK}^{3+}$ are remarkably similar-all the major bands are at the same position-which

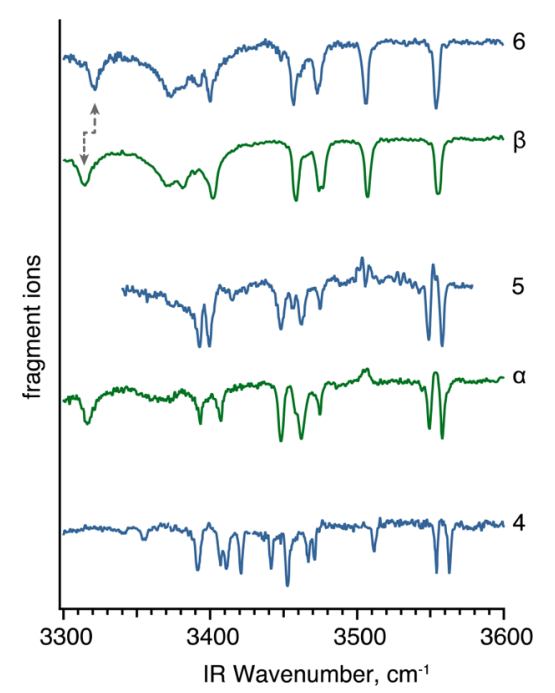

Figure 4. Comparison of vibrational spectra of conformers 4, 5, and 6 of Pro3\&7 $\rightarrow$ Ala mutant and conformers $\alpha$ and $\beta$ of nonsubstituted $\mathrm{BK}^{3+}$. The dashed line indicates the band of conformer 6 that shifts upon the mutation. We use isotopic labeling to identify this band as the serine NH stretch (Figure S1). 
suggests that these two essentially share the same structure. We expect two additional bands to appear in the $\mathrm{NH}$ stretch region of the mutant, since substituting two prolines with alanine increases the number of amide $\mathrm{NH}$ groups by two. These may be responsible for the shoulders on the peak at $3458 \mathrm{~cm}^{-1}$ or the additional intensity in the group of peaks near $3385 \mathrm{~cm}^{-1}$. The spectra of conformers 5 of the mutant and $\alpha$ (conformational family II of $\mathrm{BK}^{3+}$ ) suggest that these also share essentially the same geometry.

The vibrational spectrum (and hence the structure) of conformer 4 of the mutant is substantially different from that of any structure adopted by native $\mathrm{BK}^{3+}$, despite their overlapping $\mathrm{CV}$ distributions. The two bands in the region higher than 3550 $\mathrm{cm}^{-1}$ do not overlap with those of conformer $\alpha$, and the pattern of features in the $3380-3490 \mathrm{~cm}^{-1}$ region seems to be clearly distinct.

To summarize, we observe that the Pro $3 \& 7 \rightarrow$ Ala mutant forms two conformational families in the gas phase. One of them overlaps with conformational family II of native $\mathrm{BK}$, and both conformers $\alpha$ and $\beta$ of this family are reproduced by the mutant. This implies that prolines 3 and 7 in these two conformers of native $\mathrm{BK}$ are in the trans configuration. Conformers $1-4$ of Pro3\&7 $\rightarrow$ Ala are not accessible in nonsubstituted BK: Structures 1-3 appear at CV values higher than those recorded for $\mathrm{BK}$ and have distinct features in the electronic absorption spectrum, while conformer 4 has a distinct IR signature, which reflects substantial differences in the structure.

We compared these findings with the information obtained by drift-tube (DT) IMS. As reported by Clemmer and coworkers, ${ }^{17}$ the drift-time distribution of $\mathrm{BK}^{3+}$ has three resolved peaks, A, B and C, while the drift-time distribution of Pro3\&7 $\rightarrow$ Ala appears as one peak, which partially overlaps with family B of nonsubstituted BK (Figure S2). We preselected family B in a DT analogous to the one used by Clemmer, measuring its vibrational spectrum using $\mathrm{H}_{2}$-tagging ${ }^{26}$ and comparing it with the infrared spectra of conformers $\alpha$ and $\beta$ (Figure S4). This shows that conformational family $B$ consists mostly of conformers $\alpha$ and $\beta$. In other words, conformational family II separated by FAIMS corresponds to family B isolated by DTIMS.

Our findings imply that underneath one peak in the mutant CCS distribution there are more conformations than within family B/II of nonsubstituted BK. To exclude the possibility that they arise from collisional heating in the FAIMS device, we measured the electronic absorption spectrum of the mutant with minimal collisional activation without FAIMS separation (Figure 5). Conformations 2 and 4 are present even when the activation is minimized. Moreover, if conformers $1-4$ resulted from collisional heating, it would be possible to produce them via intentional activation in the drift tube. However, when increasing the voltage in the activation region of the DT, we do not observe any new peaks in the arrival-time distribution of the Pro3\&7 $\rightarrow$ Ala mutant (Figure S3). We conclude that an additional conformational family with similar CCS appears upon mutation.

To explain why we observe additional conformers, we turn to statistical analysis of protein structures. It is well-known that the local structure of a peptide is determined by three dihedral angles, usually denoted as $\varphi, \psi$, and $\omega$. The angle $\omega$ can take two discrete values: around $180^{\circ}$ in the trans conformation or around $0^{\circ}$ in the cis. Obviously, the conformational preferences of proline and alanine relative to other dihedral angles are also

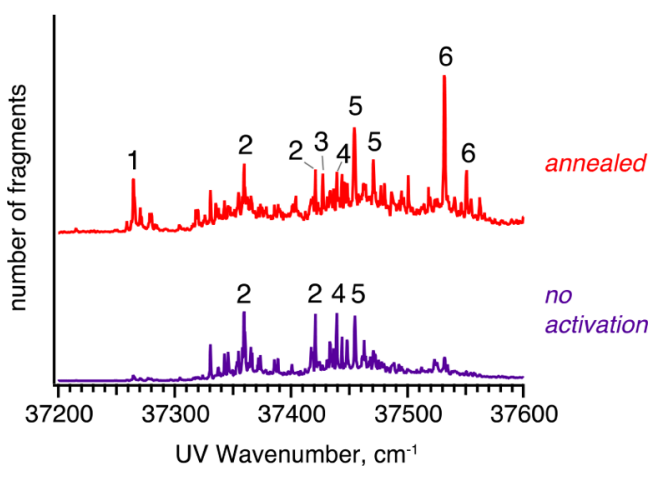

Figure 5. UV photofragmentation spectra of Pro3\&7 $\rightarrow$ Ala mutant with lowest collisional activation achievable (purple) and with high degree of collisional activation (red). The activation is controlled by the amplitude of the RF voltage applied to the ion funnel electrodes.

important. Chakrabarti and co-workers analyzed a database of 408 polypeptide chain structures and presented all torsion angles in Ramachadran plots (Figure S5). ${ }^{27}$ They clearly show that the alanine backbone can occupy a significantly larger part of the conformational space and adopt structures that are not achievable for the proline residue due to the nature of its side chain.

In the case of BK, we observe nearly identical conformations for the alanine-substituted and nonsubstituted peptide, but this does not have to be the case. It is possible that substitution of proline by alanine energetically favors structures with the torsion angles $\varphi$ and $\psi$ forbidden for proline-containing molecule. In such a case, proline-to-alanine substitution will not provide information about the cis-trans isomerization state of the native peptide. This type of behavior has been recently observed when applying mutational analysis to protein oligomerization regulation. ${ }^{28}$

Proline-to-alanine substitution has allowed us to identify two conformations of native $\mathrm{BK}^{3+}$ in the gas phase that have prolines in positions 3 and 7 in the trans configuration; thus, in this case the aim of mutational analysis could be realized. However, we find that the Pro $3 \& 7 \rightarrow$ Ala mutant does not only reproduce a part of the conformational space of $\mathrm{BK}^{3+}$, as it is often assumed, but also forms additional structures due to the higher flexibility of alanine compared to proline. This fact renders proline mutational analysis prone to inexact conclusions when used in conjunction with low-resolution structure determination techniques. Our findings can be important when using mutational analysis for elucidating the role of proline in such phenomena as transmembrane loops, intrinsically disordered proteins, or amyloidosis.

\section{ASSOCIATED CONTENT}

\section{Supporting Information}

The Supporting Information is available free of charge on the ACS Publications website at DOI: 10.1021 /jacs.7b11302.

Detailed description of our experimental approach and additional experimental data (PDF)

\section{AUTHOR INFORMATION}

\section{Corresponding Author}

*thomas.rizzo@epfl.ch

ORCID

Thomas R. Rizzo: 0000-0003-2796-905X 


\section{Notes}

The authors declare no competing financial interest.

\section{ACKNOWLEDGMENTS}

We are grateful to the EPFL, the Swiss National Science Foundation (grant no. 200020_165908) and the joint EPFLMax Planck Center for Molecular Nanotechnology for the financial support of this work.

\section{REFERENCES}

(1) Sakata, M.; Chatani, E.; Kameda, A.; Sakurai, K.; Naiki, H.; Goto, Y. J. Mol. Biol. 2008, 382, 1242-1255.

(2) Stewart, D. E.; Sarkar, A.; Wampler, J. E. J. Mol. Biol. 1990, 214, 253-260.

(3) Weiss, M. S.; Jabs, A.; Hilgenfeld, R. Nat. Struct. Mol. Biol. 1998, 5,676 .

(4) Evans, P. A.; Dobson, C. M.; Kautz, R. A.; Hatfull, G.; Fox, R. O. Nature 1987, 329, 266-268.

(5) Schultz, D. A.; Friedman, A. M.; White, M. A.; Fox, R. O. Protein Sci. 2005, 14, 2862-2870.

(6) Andreotti, A. H. Biochemistry 2003, 42, 9515-9523.

(7) Eakin, C. M.; Berman, A. J.; Miranker, A. D. Nat. Struct. Mol. Biol. 2006, 13, 202-208.

(8) Wedemeyer, W. J.; Welker, E.; Scheraga, H. A. Biochemistry 2002, 41, 14637-14644.

(9) Rousseau, F.; Schymkowitz, J. W. H.; Wilkinson, H. R.; Itzhaki, L. S. Proc. Natl. Acad. Sci. U. S. A. 2001, 98, 5596-5601.

(10) Counterman, A. E.; Clemmer, D. E. Anal. Chem. 2002, 74, 1946-1951.

(11) Glover, M. S.; Shi, L.; Fuller, D. R.; Arnold, R. J.; Radivojac, P.; Clemmer, D. E. J. Am. Soc. Mass Spectrom. 2015, 26, 444-452.

(12) Warnke, S.; Baldauf, C.; Bowers, M. T.; Pagel, K.; von Helden, G. J. Am. Chem. Soc. 2014, 136, 10308-10314.

(13) Voronina, L.; Masson, A.; Kamrath, M.; Schubert, F.; Clemmer, D.; Baldauf, C.; Rizzo, T. J. Am. Chem. Soc. 2016, 138, 9224-9233.

(14) Fort, K. L.; Silveira, J. A.; Pierson, N. A.; Servage, K. A.; Clemmer, D. E.; Russell, D. H. J. Phys. Chem. B 2014, 118, 1433614344.

(15) Morrison, L. J.; Wysocki, V. H. Int. J. Mass Spectrom. 2015, 391, $2-10$.

(16) Lietz, C. B.; Chen, Z.; Son, C. Y.; Pang, X.; Cui, Q.; Li, L. Analyst 2016, 141, 4863-4869.

(17) Pierson, N. A.; Chen, L.; Russell, D. H.; Clemmer, D. E. J. Am. Chem. Soc. 2013, 135, 3186-3192.

(18) Theillet, F.-X.; Kalmar, L.; Tompa, P.; Han, K.-H.; Selenko, P.; Dunker, A. K.; Daughdrill, G. W.; Uversky, V. N. Intrinsically Disordered Proteins 2013, 1, e24360.

(19) Williamson, M. P. Biochem. J. 1994, 297, 249-260.

(20) Hacke, M.; Gruber, T.; Schulenburg, C.; Balbach, J.; Arnold, U. FEBS J. 2013, 280, 4454-4462.

(21) Kameda, A.; Hoshino, M.; Higurashi, T.; Takahashi, S.; Naiki, H.; Goto, Y. J. Mol. Biol. 2005, 348, 383-397.

(22) Jahn, T. R.; Parker, M. J.; Homans, S. W.; Radford, S. E. Nat. Struct. Mol. Biol. 2006, 13, 195-201.

(23) Voronina, L.; Rizzo, T. R. Phys. Chem. Chem. Phys. 2015, 17, 25828-25836.

(24) Purves, R. W.; Guevremont, R. Anal. Chem. 1999, 71, 23462357.

(25) Papadopoulos, G.; Svendsen, A.; Boyarkin, O. V.; Rizzo, T. R. Faraday Discuss. 2011, 150, 243-243.

(26) Masson, A.; Kamrath, M. Z.; Perez, M. A. S.; Glover, M. S.; Rothlisberger, U.; Clemmer, D. E.; Rizzo, T. R. J. Am. Soc. Mass Spectrom. 2015, 26, 1444-1454.

(27) Chakrabarti, P.; Pal, D. Prog. Biophys. Mol. Biol. 2001, 76, 1102.

(28) Smaldone, G.; Vigorita, M.; Ruggiero, A.; Balasco, N.; Dattelbaum, J. D.; D’Auria, S.; Del Vecchio, P.; Graziano, G.;
Vitagliano, L. Biochim. Biophys. Acta, Proteins Proteomics 2016, 1864, 814-824. 